\title{
Construcción de un instrumento para valorar la actitud a la obesidad en adolescentes
}

\author{
Construction of an instrument to assess \\ the attitude to obesity in adolescents
}

\begin{abstract}
Objectives: a) To validate a scale of attitudes to obesity by teenagers, b) verify their ability for reproducibility $c$ ) propose scales for assessment according to age and sex. Subjects and methods: A descriptive study of survey type was conducted in 1252 adolescents between 12-17 years of three Municipal Facilities of the Province of Talca, Chile (584 males and 668 females). A survey was applied involving three indicators (factors): a) preference/ rejection, b) concern/disinterest) and c) will/apathy. Construct validity, internal consistency reliability (Cronbach) and scales were developed by the LMS method was evaluated. Results: Construct validity, after Varimax rotation showed 14 questions: a) preference/rejection (4 questions), b) concern/disinterest (6 questions) and c) will/apathy (4 questions). The variance for each factor was $23.5 \%, 41.0 \%$ and $24.1 \%$, respectively totaling $88.6 \%$ of explanation. The reliability showed highly reliable values, ranging from 0.73 to 0.75 for each factor. The scales developed from the LMS method showed the following cutoffs points: $p<10$ is low attitude, $\geq p 10$ to $p 90$ moderate attitude, p90 to p97 high attitude and $\geq p 97$ very high attitude to obesity. Conclusion: The results suggests that this method can be used and applied in the adolescent education system in the region of Maule, Chile, whose main purpose in making the diagnosis of possible predispositions toward obesity.
\end{abstract}

Key words: Questionnaire, attitude, obesity, adolescent validity.

\section{INTRODUCCIÓN}

En los últimos años la prevalencia de sobrepeso y obesidad se ha incrementado en niños y adolescentes y se ha convertido en una epidemia mundial (1). En la actualidad este fenómeno es un problema cada vez más común entre los adolescentes modernos (2), por lo que la preocupación por ella no sólo debe estar abocada a estudiar la prevalencia del sobrepeso y obesidad, el riesgo metabólico, la inactividad física, entre otros aspectos, sino también, se debe tomar atención en los correlatos psicosociales relacionados con la estigmatización a la obesidad entre los adolescentes.

En ese sentido, los prejuicios y la discriminación contra las personas con sobrepeso y obesidad actualmente están muy extendidos dentro de nuestra sociedad. De hecho, varios estudios demostraron que el exceso de peso genera rechazo y problemas de discriminación en diferentes ámbitos, por ejemplo, en la asistencia sanitaria (3), en el lugar de trabajo (4), en la escuela (5), en las relaciones interpersonales (6), entre otros ámbitos.

En esencia, estudiar la actitud hacia la obesidad en adolescentes es un tema de especial interés, puesto que en general, incluye creencias y sentimientos (positivos y negativos) sobre
Rossana Gómez C. (1,2,3)

Miguel de Arruda (4), Jose Sulla T. $(2,5)$

Fernando Alvear (3), Camilo Urra A. (3) Carolina González P. (3), Cristian Luarte R. (6) Marco Cossio B. $(2,7)$

(1) Universidad Autónoma de Chile, Talca, Chile. 2) Red Iberoamericana en Investigación en Desarrollo Biológico Humano, Arequipa, Perú

(3) Universidad Científica del Sur, Lima, Perú. (4) Facultad de Educación Física. Universidad Estadual de Campinas, Sao Paulo, Brasil (5) Universidad Nacional de San Agustín, Universidad Católica Santa María, Arequipa, Perú. (6) Facultad de Ciencias de la Actividad Física, Universidad San Sebastián, Concepción, Chile. (7) Departamento de Ciencias de la Actividad Física. Universidad Católica del Maule, Chile.

Dirigir la correspondencia a: Profesor

Marco A Cossio Bolaños Universidad Católica del Maule Av. San Miguel $s / n$ Talca. Chile

E-mail: mcossio1972@hotmail.com

Este trabajo fue recibido el 19 de Febrero de 2015 y aceptado para ser publicado el 30 de Julio de 2015.

las personas, objetos y eventos (7). Su valoración a menudo se efectúa por medio de técnicas de encuesta y puede ser abordada desde dos puntos de vista según Herek (8): desde la función instrumental y la función simbólica.

La función instrumental se basa en una evaluación del objeto de la actitud en términos de utilidad personal, sin embargo, la función simbólica se caracteriza por la expresión de creencias y valores, y por la manera en que el individuo quiere ser percibido por los demás. En ese contexto, son escasos los instrumentos en lengua castellana que valoren la actitud a la obesidad, aunque por lo general se utilizan algunos instrumentos propuestos por Crandall (9) y Dedeli, Fadiloglu (10).

En general, el presente estudio busca construir un cuestionario que valore la actitud hacia la obesidad basado en la función simbólica, cuyas propiedades estén relacionadas en especial con las creencias, el rechazo y la voluntad de los adolescentes.

Por lo tanto, comprender cuantitativamente los conocimientos, las actitudes, los comportamientos que rodean los patrones dietéticos y de actividad física, así como la forma y tamaño del cuerpo a través de una escala de actitudes, podría jugar un papel importante entre los adolescentes, ya que a 
través de su uso y aplicación podría identificarse grupos con baja, moderada y elevada actitud a la obesidad. A partir de ello, es posible desarrollar intervenciones orientadas a lograr cambios conductuales en la escuela, de tal forma que contribuyan a mejorar y mantener la autoestima, hacer frente a las ansiedades generadas por conflictos internos, así como valorar de forma más favorable y expresar actitudes más positivas respecto a sus estilos de vida saludables.

El presente estudio tuvo los siguientes objetivos: a) validar una escala de actitud a la obesidad para adolescentes, b) verificar su capacidad de reproductibilidad y c) proponer baremos para su valoración en función de la edad y sexo.

\section{Sujetos y Métodos}

Se efectuó un estudio de tipo descriptivo (Encuesta). La muestra seleccionada fue de tipo no-probabilística (cuotas). Se encuestó a 1252 adolescentes de ambos sexos (584 varones y 668 mujeres) de tres establecimientos Municipales de la Provincia de Talca, Chile. El rango de edad de los adolescentes oscila entre los 12 a 17 años de edad. La edad promedio de los varones fue $14,3 \pm 3$ años y de las mujeres fue $14,5 \pm 3$ años y de ambos sexos 14,4 \pm 2 años.

La muestra refleja una proporción equilibrada de participantes de acuerdo a la edad, por ejemplo, en hombres fue (16,4\% a los12 años, 21,9\% a los13 años, 15,8\% a los 14 años, $18,5 \%$ a los 15 años, $15,1 \%$ a los 16 años y 12,3\% a los 17 años). En mujeres fue del (15,0\% a los 12 años, 19,2\% a los 13 años, $22,8 \%$ a los 14 años, $18,0 \%$ a los 15 años, $14,4 \%$ a los 16 años y $10,8 \%$ a los 17 años). El tamaño y las características de la muestra estudiada se pueden observar en la tabla 1.

Se incluyeron a todos los adolescentes entre 12 a 17 años, así como a los que firmaron el consentimiento y asentimiento informado. Se excluyeron a los que no asistieron los días que se aplicó el cuestionario y a los que vivían en zona rural. El estudio contó con autorización del Comité de Etica local de la Universidad Autónoma de Chile.

\section{Procedimientos}

El instrumento se construyó a partir de la operacionalización de la variable actitud a la obesidad (tabla 2). La variable presenta tres indicadores y cada una de ellas engloba un conjunto de preguntas formuladas a partir de validez de contenido. Luego se elaboró el cuestionario considerando dos secciones: información general e información específica (preguntas de actitud a la obesidad). Las alternativas utilizadas fueron tipo Likert, en el que se indicaba: a) totalmente de acuerdo, b) ni de acuerdo ni en descuerdo y c) totalmente en desacuerdo (ver anexos).

El instrumento se aplicó a los adolescentes en horarios de clases, entre las 8.30 a 14.30 horas y durante los meses de octubre y noviembre del 2014. Se dispuso entre 15 a 20 minutos para responder las preguntas del instrumento. Todo el procedimiento de recolección de datos estuvo a cargo de 6 estudiantes de Educación Física, el cual, previamente fueron capacitados en técnicas de la encuesta. Para garantizar la comprensión de los ítems del instrumento, previamente se aplicó una prueba piloto a adolecentes de un establecimiento diferente al del estudio.

La validez del instrumento se determinó por constructo. Se utilizó la técnica de análisis factorial confirmatoria con más de 1 factor (tres factores) y la fiabilidad se verificó por medio de consistencia interna a través de alfa de Cronbach.

La propuesta de baremación se efectuó por medio de percentiles para cada sexo y edad. Los puntos de corte para la escala de actitud hacia la obesidad son: $p<10$ es baja actitud,

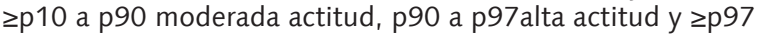
muy alta actitud hacia la obesidad. Según las afirmaciones respondidas, los resultados pueden reflejar actitudes positivas hacia la obesidad y/o interpretadas como favorables.

\section{Análisis estadístico}

Se verificó la normalidad de los datos por medio de Kolmogorov-Smirnov. Se calcularon los estadígrafos descriptivos de media $(X)$, desviación estándar (DE), frecuencias, porcentajes y distribución percentilar a través del método LMS (11) en p3, p10, p25, p50, p75, p90, y p97. Previamente los datos fueron normalizados para cada edad y se tuvo que realizar una transformación de potencia de Box-Cox. Para ello se aplicó el procedimiento de máxima probabilidad de penalización a efecto de crear tres curvas suaves: $L(t)$ Box-Cox Power, $M(t)$ mediana y $S(t)$ Coeficiente de variación. Para la validez del instrumento se utilizó el método multivariado de análisis factorial con la técnica de extracción de componentes principales, seguida de rotación Varimax y Kaiser-Meier-Olkin (KMO). La

TABLA 1

Características básicas de la muestra estudiada

\begin{tabular}{lrr}
\hline Variables & $\mathrm{n}$ & $\%$ \\
\hline Sexo: & 584 & 46,6 \\
Masculino & 668 & 53,4 \\
Femenino & 1252 & 100 \\
Total & & 37,5 \\
Actividad deportiva: & 470 & 31,2 \\
Siempre & 390 & 31,3 \\
A veces & 392 & 100 \\
Nunca & 1252 & 31,3 \\
Total & & 38,6 \\
Fuma: & 392 & 30,1 \\
Siempre & 484 & 100 \\
A veces & 376 & 1252 \\
Nunca & & \\
Total & & \\
\hline
\end{tabular}


fiabilidad se determinó por el Coeficiente a Cronbach. Los resultados fueron procesados en planillas de Excel y SPSS 18.0.

\section{Resultados}

La figura 1 muestra los valores medios \pm DE obtenidos en las 20 preguntas iníciales del instrumento elaborado. Las preguntas 4, 7, 15 y 19 fueron eliminadas por presentar saturaciones inferiores a 0,50.

El análisis factorial de componentes principales con rotación Varimax se observan en la tabla 3. El factor A (pre-
ferencias/Rechazo) agrupó 4 preguntas (1-4), mostrando 23,4\% de explicación. El factor B (Preocupación/Desinterés) agrupó 6 preguntas (5-10) y un 41\% de explicación de la varianza. El factor C (Voluntad/Apatía) reunió 4 preguntas y evidenció $24 \%$ de explicación. En general, la versión del instrumento con 14 ítems presentó 88,6\% de explicación, y consecuentemente valores superiores de saturación de 0,50 , respectivamente.

La fiabilidad del instrumento se observa en la tabla 4. Las correlaciones ítem total mostraron valores entre 0,28 a

FIGURA 1

Valores medios \pm DE de la escala de actitud a la obesidad en adolescentes.

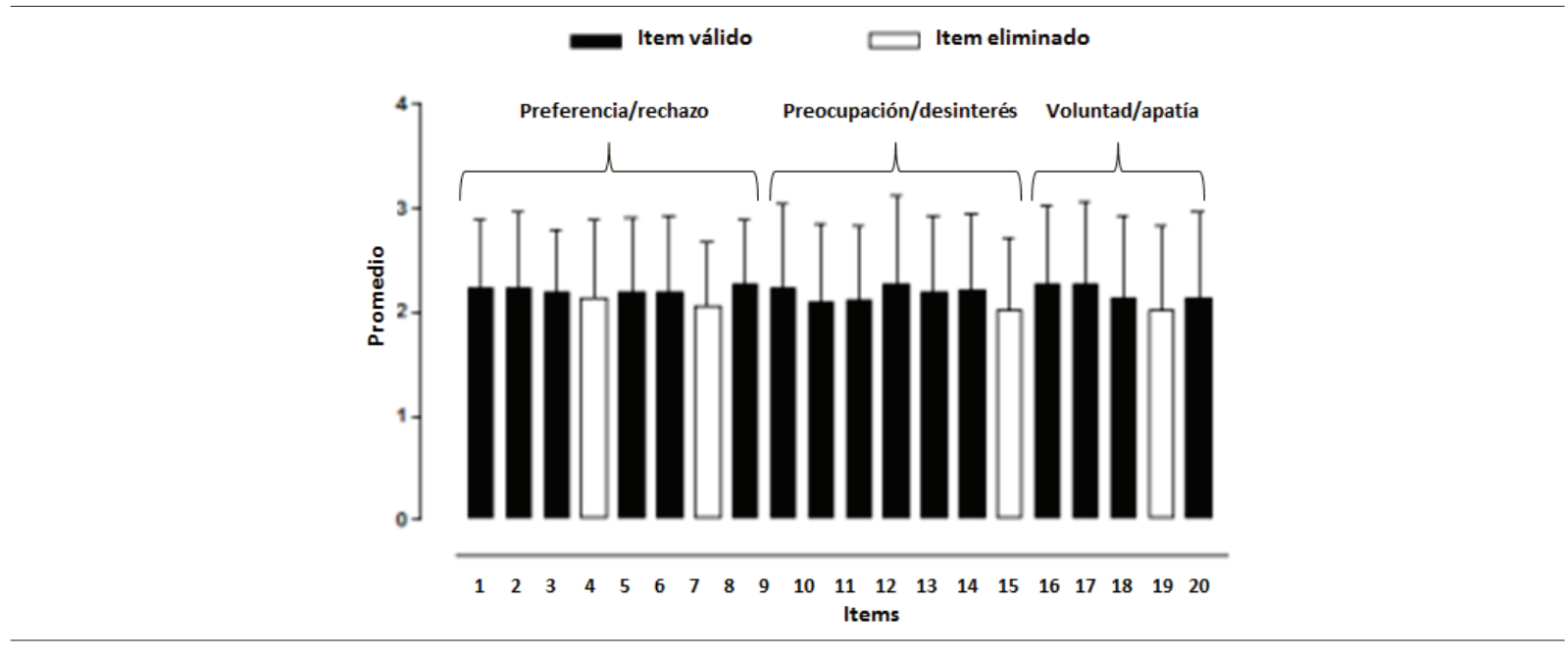

TABLA 2

Operacionalización de la variable actitud a la obesidad

\begin{tabular}{|c|c|c|}
\hline Variable & Indicadores & Sub-indicadores \\
\hline \multirow{3}{*}{ 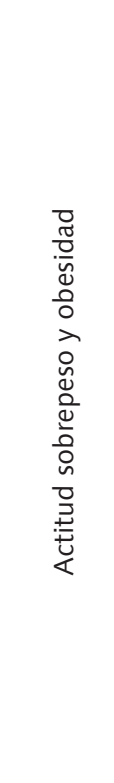 } & 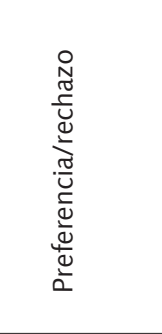 & $\begin{array}{l}\text { 1. Con frecuencia prefiero quedarme en casa, que salir a jugar al parque. } \\
\text { 2. A menudo no me llama la atención mucho hacer ejercicios físicos. } \\
\text { 3. Me agrada salir a reuniones sociales con mis amigos. } \\
\text { 4. Me siento más a gusto comiendo en la calle, que en mi casa. } \\
\text { 5. Cuando tengo tiempo libre, prefiero mirar televisión en casa. } \\
\text { 6. Cuando estoy en casa no me gusta ayudar en los quehaceres del hogar } \\
\text { (limpiar, barrer, planchar, cortar pasto, etc). } \\
\text { 7. Me agrada más la idea de comer comida rápida que las frutas } \\
\text { 8. Cuando estoy en el colegio me gusta sentarme para conversar con mis } \\
\text { amigos. }\end{array}$ \\
\hline & 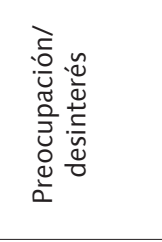 & $\begin{array}{l}\text { 9. Te sentirías preocupado en el caso de que subas de peso repentinamente? } \\
\text { 10. Alguna vez me he sentido intranquilo por mi barriga? } \\
\text { 11. Me preocupa mucho el hecho de no poder mantener mi peso ideal. } \\
\text { 12. Actualmente consideras que estás subiendo de peso? } \\
\text { 13. A menudo trato de no ingerir alimentos que me engorden. } \\
\text { 14. Me preocupo mucho cuando ingiero alimentos ricos en grasas. } \\
\text { 15. Últimamente siento que no estoy haciendo actividad física. }\end{array}$ \\
\hline & 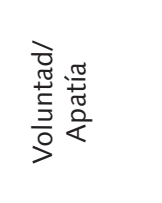 & $\begin{array}{l}\text { 16. Considero que tengo mucha voluntad para hacer ejercicios físicos. } \\
\text { 17. En general, considero que tengo autocontrol cuando ingiero mis alimentos. } \\
\text { 18. Pienso que soy capaz de mantener un estilo de vida saludable. } \\
\text { 19. Creo que puedo subir de peso si me dejo vencer por la flojera. } \\
\text { 20. Considero que los ejercicios físicos son importantes para mantener mi peso } \\
\text { corporal. }\end{array}$ \\
\hline
\end{tabular}


0,62 . El $\alpha$ Cronbach para el Factor $A$ evidenció $\alpha=0,75$, para el Factor $B \alpha=0,74$ y para el Factor $C \alpha=0,73$. Los valores de $\alpha$ de Cronbach por pregunta oscilan entre 0,72 a 0,76. Todo el instrumento muestra una confiabilidad de 0,75.

Respecto a la baremación, la tabla 5 muestra los percentiles para valorar la actitud hacia la obesidad en adolescentes en función de la edad y sexo. Se construyó utilizando el método LMS a partir de la suma de los ítems de la escala (sumatoria total de los puntajes obtenidos). Los valores de la mediana para ambos sexos son ascendentes conforme la edad avanza.

\section{Discusión}

Los procedimientos o técnicas estadísticas que más destacan para contrastar la validez de constructo son el análisis factorial exploratorio AFE y el análisis factorial confirmatorio AFC. Este estudio utilizó el AFC, puesto que se basó en teorías que dirigen la propia definición operacional indicando tres sub-

\section{TABLA 3}

Análisis factorial de los ítems tras rotación Varimax.

$\mathrm{N}^{\circ}$ ítems

A) Preferencia/Rechazo

1 Me agrada salir a reuniones sociales con mis amigos.

2 Cuando tengo tiempo libre, prefiero mirar televisión en casa.

3 Cuando estoy en casa no me gusta ayudar en los quehaceres del hogar (limpiar, barrer, planchar, cortar pasto, etc).

4 Cuando estoy en el colegio me gusta sentarme para conversar con mis amigos.

B) Preocupación/Desinterés

5 Te sentirías preocupado en el caso de que subas de peso repentinamente?

6 Alguna vez me he sentido intranquilo por mi barriga?

7 Me preocupa mucho el hecho de no poder mantener mi peso ideal.

8 Actualmente consideras que estás subiendo de peso?

9 A menudo trato de no ingerir alimentos que me engorden.

$10 \mathrm{Me}$ preocupo mucho cuando ingiero alimentos ricos en grasas.

C) Voluntad/Apatía

11 Considero que tengo mucha voluntad para hacer ejercicios físicos.

12 En general, considero que tengo autocontrol cuando ingiero mis alimentos.

13 Pienso que soy capaz de mantener un estilo de vida saludable.

14 Considero que los ejercicios físicos son importantes para mantener mi peso corporal.
Factores

B

C
0,56
0,66
0,56
0,58

\begin{tabular}{|c|c|c|c|}
\hline $\begin{array}{l}\text { Valores propios } \\
\% \text { de explicación de la Varianza }\end{array}$ & $\begin{array}{l}2,30 \\
23,50\end{array}$ & $\begin{array}{c}4,10 \\
41,00\end{array}$ & $\begin{array}{l}2,40 \\
24,10\end{array}$ \\
\hline
\end{tabular}

Leyenda: $\mathrm{KMO}=0,64 \times 2=525,3, \mathrm{p}=0,000$.

\section{TABLA 4}

Valores de Confiabilidad Ítem-total y alfa de Cronbach del instrumento.

$\mathrm{N}^{\circ} \quad$ Ítems

\section{A) Preferencia/Rechazo}

1 Me agrada salir a reuniones sociales con mis amigos.

2 Cuando tengo tiempo libre, prefiero mirar televisión en casa.

3 Cuando estoy en casa no me gusta ayudar en los quehaceres del hogar (limpiar, barrer, planchar, cortar pasto, etc).

4 Cuando estoy en el colegio me gusta sentarme para conversar con mis amigos.

B) Preocupación/Desinterés

5 Te sentirías preocupado en el caso de que subas de peso repentinamente?

6 Alguna vez te has sentido intranquilo por tu barriga?

7 Me preocupa mucho el hecho de no poder mantener mi peso ideal.

8 Actualmente consideras que estás subiendo de peso?

9 A menudo trato de no ingerir alimentos que me engorden.

10 Me preocupo mucho cuando ingiero alimentos ricos en grasas.

C) Voluntad/Apatía

11 Considero que tengo mucha voluntad para hacer ejercicios físicos.

12 En general, considero que tengo autocontrol cuando ingiero mis alimentos.

13 Pienso que soy capaz de mantener un estilo de vida saludable.

14 Considero que los ejercicios físicos son importantes para mantener mi peso corporal.
Correlación Elemento Total

0,78

0,70

0,58

0,70 
indicadores (Preferencia/Rechazo, Preocupación/desinterés y Voluntad/Apatía). Esta operacionalización está respaldada por la correspondencia teórica entre el constructo y el dato, como lo sugiere Schmitt (12).

En esencia, el instrumento desarrollado y propuesto presentó un elevado poder de explicación $(88,6 \%)$, inclusive los valores propios son superiores a 1 de acuerdo a lo recomendado por Kaiser (13). Estos resultados evidencian un elevado nivel de validez al igual que otros estudios que valoran escalas de actitud hacia la obesidad $(9,10,14)$, independientemente del tipo de muestra y rango de edad. Además otros estudios que investigaron la validez de constructo en temáticas relacionadas al presente estudio, reportaron valores de varianza y cargas factoriales similares y hasta inferiores al presente estudio (15-17).

En ese sentido, la aproximación deductiva y/o confirmatoria de los ítems propuestos para cada indicador de la variable actitud hacia la obesidad, en su mayoría fueron válidas, excepto las preguntas 4, 7, 15 y 19 que fueron eliminadas, debido a los bajos valores observados en sus cargas factoriales (inferiores a 0.40), a pesar de que los estudios en general consideran como válidos, valores superiores a 0,40 (10,15,18). De hecho, la estructura obtenida a partir del AFC, en su mayoría

TABLA 5

Valores LMS y distribución percentilar para valorar la actitud a la obesidad en adolescentes escolares.

\begin{tabular}{|c|c|c|c|c|c|c|c|c|c|c|c|}
\hline Edad (años) & $\mathrm{n}$ & L & $M$ & $S$ & P3 & P10 & P25 & P50 & P75 & P90 & P97 \\
\hline \multicolumn{12}{|l|}{ Hombres } \\
\hline 12 & 96 & 1,3 & 29,1 & 0,1 & 20,3 & 23,4 & 26,3 & 29,1 & 31,8 & 34,4 & 37 \\
\hline 13 & 128 & 2,2 & 30,3 & 0,1 & 21,5 & 24,8 & 27,8 & 30,3 & 32,7 & 34,8 & 36,8 \\
\hline 14 & 92 & 0,9 & 30,2 & 0,2 & 21,1 & 24,1 & 27,1 & 30,2 & 33,3 & 36,4 & 39,5 \\
\hline 15 & 108 & 1,1 & 33,8 & 0,1 & 23,9 & 27,2 & 30,5 & 33,8 & 37,0 & 40,3 & 43,5 \\
\hline 16 & 88 & 1,4 & 35,8 & 0,1 & 26,6 & 29,8 & 32,9 & 35,8 & 38,7 & 41,4 & 44,1 \\
\hline 17 & 72 & 2,0 & 33,8 & 0,1 & 27,0 & 29,5 & 31,7 & 33,8 & 35,8 & 40,7 & 44,1 \\
\hline \multicolumn{12}{|l|}{ Mujeres } \\
\hline 12 & 100 & 0,9 & 32,1 & 0,2 & 18,8 & 23,2 & 27,6 & 32,1 & 36,6 & 41,2 & 45,9 \\
\hline 13 & 128 & 0,6 & 31,6 & 0,2 & 20,2 & 23,8 & 27,6 & 31,6 & 35,8 & 40,2 & 44,9 \\
\hline 14 & 152 & 0,5 & 33,1 & 0,2 & 22,5 & 25,8 & 29,4 & 33,1 & 37,0 & 41,2 & 45,5 \\
\hline 15 & 120 & 0,5 & 37,4 & 0,1 & 27,7 & 30,8 & 34,0 & 37,4 & 41,0 & 44,7 & 48,5 \\
\hline 16 & 96 & 0,1 & 40,1 & 0,1 & 32,6 & 35,0 & 37,5 & 40,1 & 43,0 & 46,1 & 49,3 \\
\hline 17 & 72 & 0,1 & 40,7 & 0,1 & 35,1 & 36,8 & 38,7 & 40,7 & 42,8 & 46,2 & 47,6 \\
\hline
\end{tabular}

Leyenda: * diferencia significativa en relación a las mujeres $(p<0,05)$, L= asimetría; $M=$ mediana; $S=$ coeficiente de variación.

\section{ANEXO}

\section{CUESTIONARIO DE ACTITUD A LA OBESIDAD}

Nombre:

Fecha de Nacimiento: día ( ) Mes ( ) Año ( ). Sexo: (M) (F). Zona geográfica donde vive: Urbana ( ) Rural ( ). Grado de Instrucción padre/madre: Analfabeto ( ), Escolar ( ), Superior ( ). Formación académica padres: Profesional ( ), Técnica ( ) Oficio ( ). Estado actual de Ocupación padres: Desempleado ( ), Empleado ( ). Fuma: Si ( ) No( )

Marca con una equis $(x)$ la respuesta que consideres. Se acepta marcar una sola alternativa.

Leyenda: TA: Totalmente de acuerdo, N.D.N.D: Ni de acuerdo, ni en desacuerdo

T.D: Totalmente en descuerdo

T.A N.D.N.D T.D

1. Me agrada salir a reuniones sociales con mis amigos.

(3) (2)

(3) (2)

(1)

2. Cuando tengo tiempo libre, prefiero mirar televisión en casa.

(2) (1)

3. Cuando estoy en casa no me gusta ayudar en los quehaceres del hogar (limpiar, barrer, planchar, cortar pasto, etc.).

4. Cuando estoy en el colegio me gusta sentarme para conversar con mis amigos.

(3) (2)

6. Alguna vez te has sentido intranquilo por tu barriga?

7. Me preocupa mucho el hecho de no poder mantener mi peso ideal.

9. A menudo trato de no ingerir alimentos que me engorden.

10. Me preocupo mucho cuando ingiero alimentos ricos en grasas.

12. En general, considero que tengo autocontrol cuando ingiero mis alimentos.

13. Pienso que soy capaz de mantener un estilo de vida saludable.

14. Considero que los ejercicios físicos son importantes para mantener mi peso corporal.
(2)

(1) 
es coincidente con la operacionalización teórica propuesta inicialmente, por lo que el instrumento propuesto presentó un modelo razonable y una validez de constructo satisfactoria. Evidentemente, la literatura respalda que el modelo AFC corrige las deficiencias inherentes a la perspectiva exploratoria, por lo que una de sus potencialidades es conducir principalmente a la contrastación de teorías sustantivas $(19,20)$, con lo cual es posible confirmar el modelo propuesto, como se verificó en el presente estudio.

En términos de calidad de medición, la confiabilidad es un requisito necesario que se debe efectuar después del proceso de validación. Su propósito principal según Goto, Mascie-Taylor (21) es alcanzar una mayor precisión y capacidad de reproductibilidad de los instrumentos. En ese sentido, la literatura reporta tres procedimientos usuales, como el test y re-test, mitades partidas y consistencia interna (22).

El presente estudio optó por la consistencia interna ( $\alpha$ Cronbach), donde los resultados evidenciaron para los tres sub-indicadores (factores) altos valores de confiabilidad. El primer indicador Preferencia/Rechazo mostró 0,75, el segundo indicador Preocupación/Desinterés fue de 0,74 y el tercer indicador Voluntad/Apatía evidenció 0,73.

En general, el instrumento en su totalidad presentó un $\alpha$ de Cronbach de 0,75 , similar a algunos estudios $(23,24)$ y relativamente inferiores a otras escalas $(10,25)$, aunque en esencia, los valores de consistencia interna observados en este estudio son moderados según lo descrito por Oviedo, Campos (26), en el que destacan valores aceptables entre 0,70 a 0,90. Además este coeficiente a menudo es el que más se utiliza para verificar la confiabilidad de los instrumentos, inclusive está directamente relacionado con las saturaciones de los indicadores (factores) estudiados (27), lo que viabiliza su uso y aplicación dentro del proceso de validez y confiabilidad en estudios por encuesta.

En cuanto a la baremación, el presente estudio buscó proponer percentiles para comparar el resultado obtenido de un individuo con los resultados de la muestra estudiada. De hecho, esta propuesta permitirá clasificar a los adolescentes escolares, con lo cual, es posible comprender de mejor forma los procesos generales de perjuicio y/o de identificar a los adolescentes con actitud favorable hacia la obesidad.

En ese sentido, la baremación tiende a apoyar en la identificación de los adolescentes con mayor o menor actitud hacia la obesidad, dado que las actitudes pueden jugar un papel importante en el mantenimiento de la autoestima (28) y en la adopción de estilos de vida nocivos y saludables. De hecho, los resultados obtenidos por medio de la baremacion propuesta pueden ser utilizados para formular estrategias de intervención (29), principalmente en aquellos adolescentes que se encuentren con elevada actitud a la obesidad.

La nueva escala de actitudes desarrollada y propuesta por la Red Iberoamericana de Investigación en Desarrollo Biológico Humano, representa una herramienta válida y confiable y sirve para diagnosticar la predisposición de los adolescentes hacia la obesidad, al menos en la región del Maule (Chile).

Su aplicación y uso está orientada a la atención clínica y a la masificación dentro del sistema escolar, puesto que en la actualidad la obesidad es considerada en gran parte como un problema de comportamiento (30) y de salud pública (31), el cual, va en aumento no sólo en Chile (32), sino a nivel mundial, por lo tanto, el instrumento propuesto podría traducirse en una herramienta fundamental para determinar las actitudes hacia la obesidad en contextos transversales y/o longitudinales. Posteriormente podría optarse por aplicar en diversas propuestas y estrategias para promocionar los hábitos de vida saludables y la práctica de la actividad física $(33,34)$, cuyos objetivos reales podrían contribuir a la reducción de los elevados índices de actitud a la obesidad.

Varias potencialidades deben ser rescatadas a partir del instrumento elaborado. Por ejemplo, permite valorar la actitud hacia la obesidad en función de la edad y sexo, y en términos de aplicabilidad se puede realizar de forma rápida, sencilla y con bajo coste, e inclusive entrega el resultado de forma inmediata (on-line) a los encuestados. Además, a diferencia de otros instrumentos, esta escala, permite identificar a los adolescentes que podrían presentar mayor o menor actitud hacia la obesidad y a partir de ello generar políticas públicas de acuerdo a su realidad. Por otro lado, algunas limitaciones deben ser consideradas, puesto que la selección de la muestra fue de tipo no-probabilística (accidental) y el rango de edad abarca desde los 12 hasta los 17 años, por lo que su aplicación se limita a adolescentes escolares de ambos sexos y de la provincia de Talca, Chile.

En conclusión, la nueva Escala de Actitudes hacia la obesidad propuesta para adolescentes demostró validez de constructo satisfactoria y moderado nivel de consistencia interna. Los resultados sugieren su uso y aplicación en adolescentes escolares del sistema educativo de la región del Maule de Chile. Este instrumento puede ser utilizado en la escuela para diagnosticar la actitud hacia la obesidad en el siguiente link: http://reidebihu.net/cuestionario.php

\section{RESUMEN}

Objetivos: a) validar una escala de actitud a la obesidad para adolescentes, b) verificar su capacidad de reproductibilidad y c) proponer baremos para su valoración en función de la edad y sexo. Sujetos y métodos: Se efectuó un estudio de tipo descriptivo por encuesta. Se encuestó a 1252 adolescentes entre 12 a 17 años de tres Establecimientos Municipales de la Provincia de Talca, Chile (584 varones y 668 mujeres). Se aplicó un cuestionario con tres indicadores (factores): a) preferencia/ rechazo, b) preocupación/desinterés y c) voluntad/apatía. Se evaluó la validez de constructo, la fiabilidad por consistencia interna (Cronbach) y los baremos fueron desarrollados por medio del método LMS. Resultados: La validez de constructo, tras la rotación Varimax evidenció 14 preguntas: a) preferencia/rechazo (4 preguntas), b) preocupación/desinterés (6 preguntas) y c) voluntad/apatía (4 preguntas). El porcentaje de explicación de la varianza para cada factor fue 23,5\%, 41,0\% y $24,1 \%$, totalizando un $88,6 \%$ de explicación. La fiabilidad evidenció valores altamente confiables, oscilando entre 0,73 a 0,75 para cada factor. Los baremos desarrollados a partir del método LMS, mostraron los siguientes puntos de corte: $p<10$ es baja actitud, $\geq$ p10 a p90 moderada actitud, p90 a p97alta actitud $y \geq p 97$ Muy alta actitud a la obesidad. Conclusión: El instrumento elaborado mostró validez de constructo satisfactoria y moderado nivel de consistencia interna. Los resultados sugieren su uso y aplicación en adolescentes escolares del sistema educativo de la región del Maule de Chile. Este instrumento puede ser utilizado en la escuela para diagnosticar posibles predisposiciones hacia la obesidad.

Palabras clave: Cuestionario, actitud, obesidad, adolescentes, validez.

\section{Agradecimientos}

Este estudio fue financiado por el Fondo Nacional de Desarrollo Científico Tecnológico y de Innovación Tecnológica (FONDECYT \# 1141295). 


\section{BIBLIOGRAFÍA}

1. Lobstein T, Baur L, Uauy R, IASO International Obesity Task Force: Obesity in children and young people: a crisis in public health. Obes Rev. 2004;1 (Suppl):4-104.

2. Klaczynski PA, Goold KW, Mudry JJ. Culture, Obesity Stereotypes, Self-Esteem, and the "Thin Ideal": A Social Identity Perspective. J Youth Adolesc. 2004; 33(4):307-17.

3. Hebl MR, Xu J. Weighing the care: Physicians' reactions to the size of a patient. International J Obes. 2001;25(8):1246-52.

4. Roehling MV, Roehling PV, Pichler S. The relationship between body weight and perceived weight-related employment discrimination: The role of sex and race. $J$ Vocational Behav. 2007; 71(2):300-18.

5. Hayden-Wade HA, Stein RI, Ghaderi A, Saelens BE, Zabinski M, Wilfley DE. Prevalence, characteristics, and correlates of teasing experiences among overweight children vs. non-overweight peers. Obesity Res. 2005;13(8):1381-92.

6. Falkner $N H$, Neumark-Sztainer D, Story $M$, Jeffery $R W$, Beuhring T, Resnick MD. Social, educational and psychological correlatesof weight status in adolescents. Obes Res. 2001; 9(1):32-42.

7. Schneider DJ. Introduction to Social Psychology, Harcourt Brace Jovanovich, New York;1988.

8. Herek GM. Can functions be measured? A new perspective on the functional approach to attitudes. Social Psychol Quart. 1987;50:285-303.

9. Crandall Ch. Prejudice Against Fat People: Ideology and Self-Interest. J Personality Social Psychol. 1994;66(5):88294.

10. Dedeli O, Fadiloglu C. Development and evaluation of the Health Balief Model Scale in obesity. TAF Preventive Med Bull. 2011; 10(5):533-42.

11. Cole TJ, Bellizzi MC, Flegal KM, Dietz WH. Establishing a standard definition for child overweight and obesity worldwide: international survey. BMJ 2000;320:1240-3.

12. Schmitt FF. Truth: A primer. Boulder, Colorado:Westviw Press; 1995.

13. Kaiser HF. The application of electronic computers to analysis factorial. Educ Psychol Mea.1960;20:141-51.

14. Lewis RJ, Cash TF, Jacobi L, Bubb-Lewis C. Prejudice toward fat people: the development and validation of the anti-fat attitudes test. Obes Res. 1997;5:297-307.

15. Unikel C, Gómez-Peresmitré G. Validez de constructo de un instrumento para la detección de factores de riesgo en los trastornos de la conducta alimentaria en mujeres mexicanas. Salud Mental. 2004;27(1); 38-49.

16. Pino JL, Díaz C, López MA. Construcción y validación de un cuestionario para medir conductas y hábitos alimentarios en usuarios de la atención primaria de salud. Rev Chil Nutr. 2011;38(1); 41-51.

17. Lima-Serrano M. Lima-Rodríguez J, Sáez-Bueno A. Diseño y validación de dos escalas para medir la actitud hacia la alimentación y la actividad física durante la adolescencia. Rev Esp Salud Pública.2012; 86:253-68.

18. Tomás JM, Oliver A. Análisis Psicométrico Confirmatorio de una Medida Multidimensional del Autoconcepto en
Español. R. interam.Psicol.2004;38(2):285-93

19. Batista-Foguet JM, Coenders G, Alonso J. Análisis factorial confirmatorio. Su utilidad en la validación de cuestionarios relacionados con la salud. Med Clin (Barc). 2004;122(Sup/ 1):21-7.

20. Pérez-Gil JA, Chacón Moscoso S, Moreno Rodríguez R. Validez de constructo: el uso de análisis factorial exploratorio-confirmatorio para obtener evidencias de validez. Psicothema. 2000;12 (Supl. 2): 442-6.

21. Goto R, Mascie-Taylor NCG. Precision of measurement as a component of human variation. J PhysiolAnthropol. 2007; 26: 253-6.

22. Thomas JR, Nelson JK. Métodos de pesquisa em atividade física. Editora Phorte, São Paulo; 2002.

23. Allison DB, Basile VC, Yuker HE. The measurement of attitudes toward and beliefs about obese person. Internat J Eating Dis. 1991;10(5):599-607.

24. Gago Sampedro J, Tornero Quiñones I, Carmona Márquez J, Sierra Robles A. Actitudes hacia la obesidad de los estudiantes de Magisterio de la Universidad de Huelva. Retos. Nuevas tendencias Educación Física, Deporte Recreación 2012;22:38-42.

25. Dedeli O, Aybarc S, Bursalioglu S, Deveci A. Validity and reliability of the Turkish version of the attitudes toward obese persons scale and the beliefs about obese persons scale. Clin Nursing Studies. 2014;2(4):105-17.

26. Oviedo C, Campo-Arias A. Aproximación al uso del coeficiente alfa de Cronbach. Rev Colomb Psiquiatr. 2005:34:572-80.

27. Cortina JM. What is coefficient alpha? En examination of theory and applications.J Appl Psychol 1993;78:98-104

28. Shavitt S. Operationalizing functional theories ofattitudes. In A. R. Pratkanis, S. J. Breckler, \& A. G.Greenwald (Eds.), Attitude structure and function. Hillsdale, NJ: Lawrence Erlbaum Associates; 1989.p.311-37.

29. Gordon-Larsen P. Obesity-Related Knowledge, Attitudes, and Behaviors in Obese and Non-obese Urban Philadelphia Female Adolescents. Obes Res. 2001; 9(2):112-8.

30. Foster D, Thomas A, Wadden A, Davidson D, Sanderson $R$, Allison D, Kessler A. Primary care physicians' attitudes about obesity and its treatment. Obes Res. 2003;11:116877.

31. Cano Garcinuno A, PerezGarcia I, Casares Alonso I. Obesidad infantil: opiniones y actitudes de los pediatras. Gac Sanit. 2008;22(2):98-104.

32. Sistema de Medición de la Calidad de la Educación del Ministerio de Educación de Chile (SIMCE, 20012). Informe de resultados de educación física $8^{\circ}$ básico, Ministerio de Educación, Unidad de currículum y evaluación, Santiago, 2011.

33. Lobo F. Políticas públicas para la promoción de la alimentación saludable y la prevención de la obesidad. Rev Esp Salud Pública. 2007; 81(5):437-41.

34. Ballesteros JM, Dal-Re M, Pérez-Farinós N, Villar C. La estrategia para la nutrición, actividad física y prevención de la obesidad (estrategia NAOS). Rev Esp Salud Pública. 2007;81(5):443-9. 